\title{
Luxación recidivante de prótesis total de cadera
}

\author{
A. Murciaa, L.M. Azorín ${ }^{b}$, A. Blanco ${ }^{c}$, H. Ferrer ${ }^{d}$, X. Gallarte, E.García-Cimbrelo ${ }^{f}$ y S. Suso ${ }^{d}$ \\ aHospital de Cabueñes. Gijón. Asturias. España. \\ bHospital de Traumatología de la Vall d'Hebron. Barcelona. España. \\ ‘Hospital General Yagüe-Divino Vallés. Burgos. España. \\ 'Hospital Mutua de Terrassa. Terrasa. Barcelona. España. \\ eHospital Clínic. Barcelona. España. \\ ${ }^{f}$ Hospital La Paz. Madrid. España.
}

Más del $50 \%$ de los casos de caderas inestables se pueden resolver mediante la reducción cerrada de la luxación. Un intento sistemático para entender la causa de la inestabilidad, normalmente suele conducir a un tratamiento eficaz del problema cuando hace falta cirugía. Los componentes constreñidos o de retención han mejorado las posibilidades de resolver los problemas difíciles de inestabilidad, pero los efectos negativos potenciales de estos componentes deben tenerse también en cuenta. Los implantes tripolares, cuyos resultados clínicos en la literatura son muy alentadores, ocupan un lugar de elección en el arsenal terapéutico de la inestabilidad protética.

Palabras clave: cadera, prótesis, luxación, recidivante.

\section{Recurrent dislocation in total hip replacement}

Over $50 \%$ of unstable hip cases can be solved by carrying out a closed reduction of the dislocation. A systematic attempt to understand the cause of the instability, normally results in an efficient treatment of the condition in cases where surgery is required. Constrained - also known as retentive - components have enhanced the possibilities of addressing difficult instability problems, although the potential negative effects of these implants also need to be considered. Tripolar implants, whose clinical results are very encouraging according to the literature, should occupy a prominent place among the therapeutic options available for prosthetic instability.

Key words: hip, prosthesis, dislocation, recurrent.
La artroplastia total de cadera (ATC) constituye uno de los mayores éxitos de la cirugía ortopédica, y debe su desarrollo al impulso de sir John Charnley. A pesar de sus excelentes resultados, que sobrepasan el $90 \%$ a los 15 años (en el momento actual hay series con más de 30 años $^{1}$ ), es una técnica que no está exenta de complicaciones, y una de las más importantes es la luxación protésica (fig. 1). Es un problema difícil que deja al paciente preocupado con su cadera protetizada, al cirujano que ve en entredicho su reputación y a la Seguridad Social expuesta a un sobrecoste considerable.

Determinar la verdadera prevalencia de la luxación postoperatoria es difícil debido a que se trata de una com-

\footnotetext{
Correspondencia:

A. Murcia Mazón.

Servicio de COT. Hospital de Cabueñes.

Apartado 205. 33394 Gijón. Asturias.
}

Recibido: agosto de 2005.

Aceptado: septiembre de 2005. plicación multifactorial y que no disminuye con el paso de los años. Existen publicaciones que recogen series numerosas en las que se sugiere que es una complicación que se presenta entre el 0,3 y el $3 \%$ de los pacientes ${ }^{2}$, aunque las cifras que se manejan habitualmente en la literatura son muy diversas, situándose entre el 1 y el $10 \%$. Si la cirugía es primaria el porcentaje oscila entre el 0,3 y el $5 \%$, mientras que si trata de cirugía de revisión aumenta entre el 5 y el $10 \%$.

Una revisión de las compañías de seguros americanas relacionan la frecuencia de luxaciones protésicas con el número de cirugías que realiza el cirujano al año, estableciendo que si lleva a cabo menos de 5 intervenciones en este período de tiempo la frecuencia es del 4,2\%, mientras que el cirujano que realiza más de 50 artroplastias al año tiene una tasa del $1,5 \%{ }^{3}$.

La prevalencia de la luxación recidivante es muy variable en la literatura, estableciéndose alrededor del 33\% $\%^{4,5}$. El problema de las luxaciones recidivantes es difícil, y con frecuencia es necesaria una reintervención quirúrgica, que 


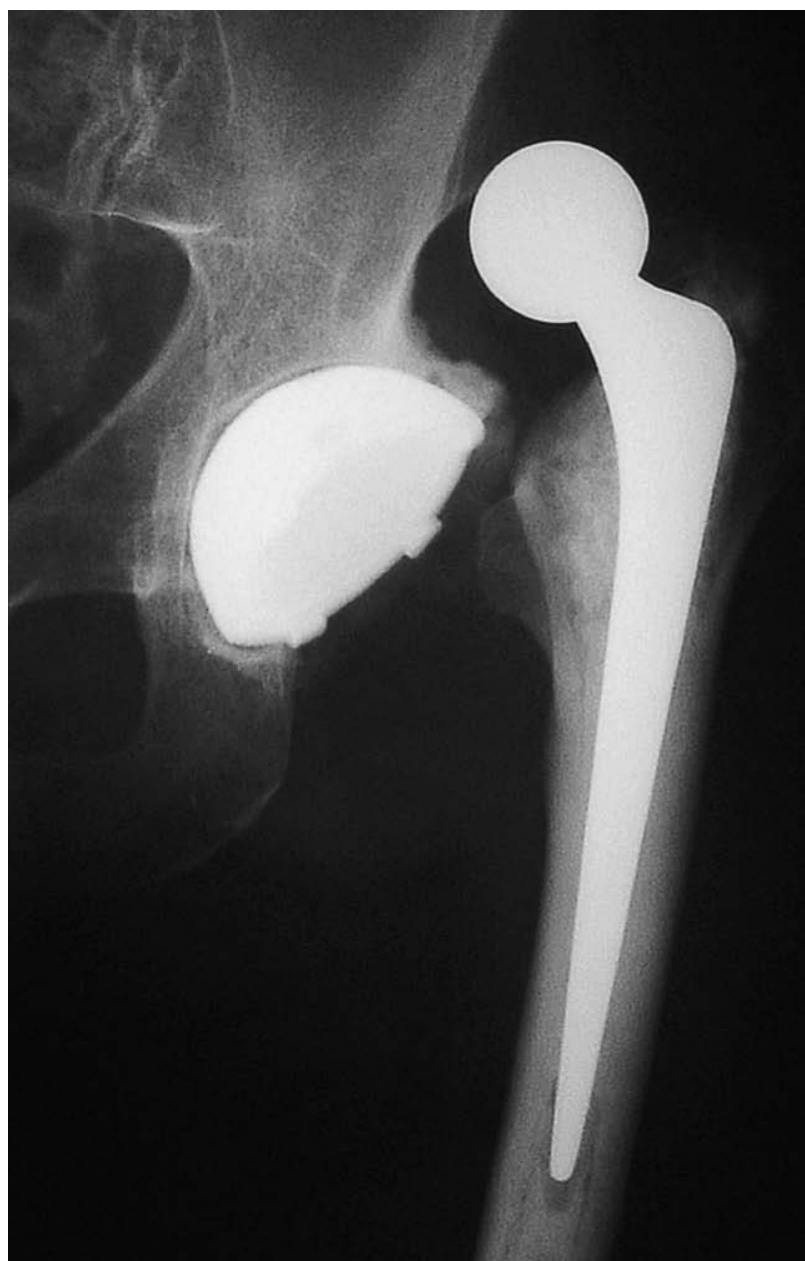

Figura 1. Artroplastia de cadera luxada.

por otro lado sólo permite restablecer la estabilidad de la articulación en un 70\% de los casos. La repercusión funcional de estos fracasos es considerable para el paciente, sobre todo en el desarrollo de la marcha, y además con manifestaciones de ansiedad y aprensión en sus actividades cotidianas. A todo ello hay que añadir la alteración del par de fricción, que conducirá a una usura prematura del implante.

El momento de la luxación se considera un factor importante. Se ha descrito una tasa de recurrencia del $60 \%$ después de una luxación tardía y una tasa del $40 \%$ después de las luxaciones que se producen en las 5 primeras semanas tras la cirugía ${ }^{6,7}$.

La inestabilidad hay que recordar que: a) no es un problema antes de la artroplastia, sino que aparece como consecuencia de ésta y que puede deberse a una técnica quirúrgica deficiente, al diseño del implante y/o al fallo en la fijación del mismo; b) produce una gran incapacidad y con frecuencia precisa cirugía adicional; c) de todos los procedimientos quirúrgicos realizados en la cadera, la reintervención por inesta- bilidad, probablemente, es la más impredecible en términos de satisfacción, tanto para el paciente como para el cirujano ${ }^{8}$.

\section{PREVENCIÓN}

El mejor tratamiento de la inestabilidad de cadera es su prevención. Para ello hay que tener en cuenta una serie de medidas que abarcan desde la planificación quirúrgica hasta los cuidados y medidas que debemos aconsejar a los pacientes una vez implantada la prótesis.

\section{Medidas preoperatorias}

Son las primeras que hay que tener en cuenta y consisten en realizar una correcta planificación con las plantillas (figs. 2 y 3), con el fin de reproducir el acodo lo más exactamente posible, el centro de rotación de la cadera y la longitud del miembro ${ }^{9-12}$. Los pasos conocidos, pero que conviene recordar, son los siguientes:

Radiografías necesarias:

1. Anteroposterior de pelvis con el foco en la sínfisis pubis.

2. Anteroposterior de la cadera a intervenir y que se vea $20 \mathrm{~cm}$ de fémur.

3. Proyección lateral o axial.

Pasos:

1. Datos del paciente, diagnóstico, lado a intervenir y tipo de implante.

2. Sobre la radiografía anteroposterior (Rx AP) de pelvis se traza una línea horizontal que una ambas tuberosidades isquiáticas, comprobando que comprende ambas corticales femorales.

3. Marcar en la misma Rx el punto más proximal de ambos trocánteres menores.

4. Medir la distancia desde el punto proximal del trocánter menor a la línea biisquiática. Cuanto mayor sea la distancia más corta estará la pierna. Si se presenta esta situación hay que anotar la diferencia de longitud de los miembros inferiores.

5. Sobre la radiografía anteroposterior de la cadera se determina el tamaño apropiado, la posición y componente acetabular con las plantillas; después se marca el centro de rotación de la cadera.

Desde el punto de vista práctico, una planificación quirúrgica sería:

1. Nombre, apellidos y número de historia.

2. Edad y sexo.

3. Diagnóstico, tipo de implante cementado (sí/no, mixto).

4. Diferencia, si existe, de longitud de los miembros inferiores (MMII).

5. Cotilo: diámetro en $\mathrm{mm}$, hemisférico, monobloque, perforado, insertos polietileno, cerámica, metal, tamaño interior. 

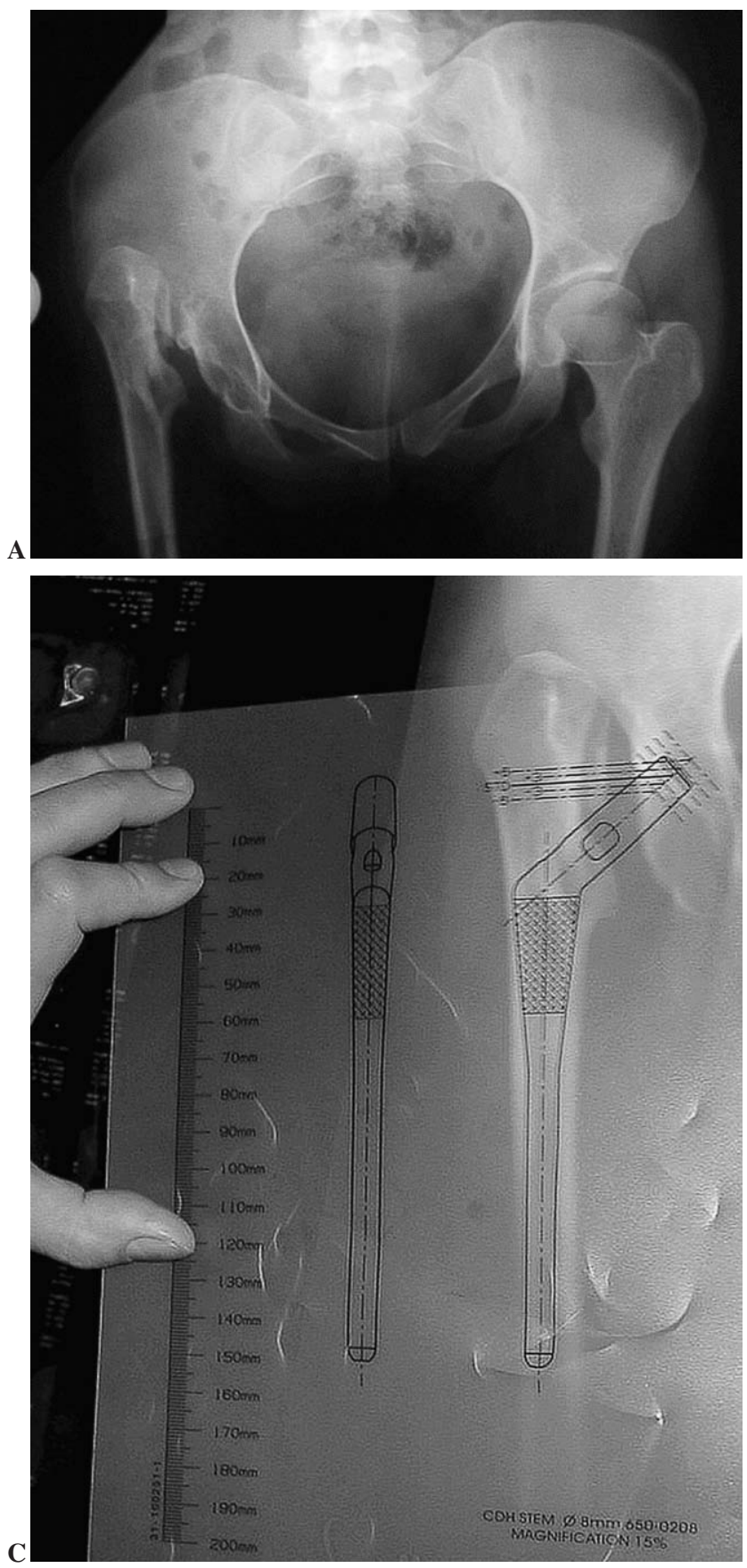

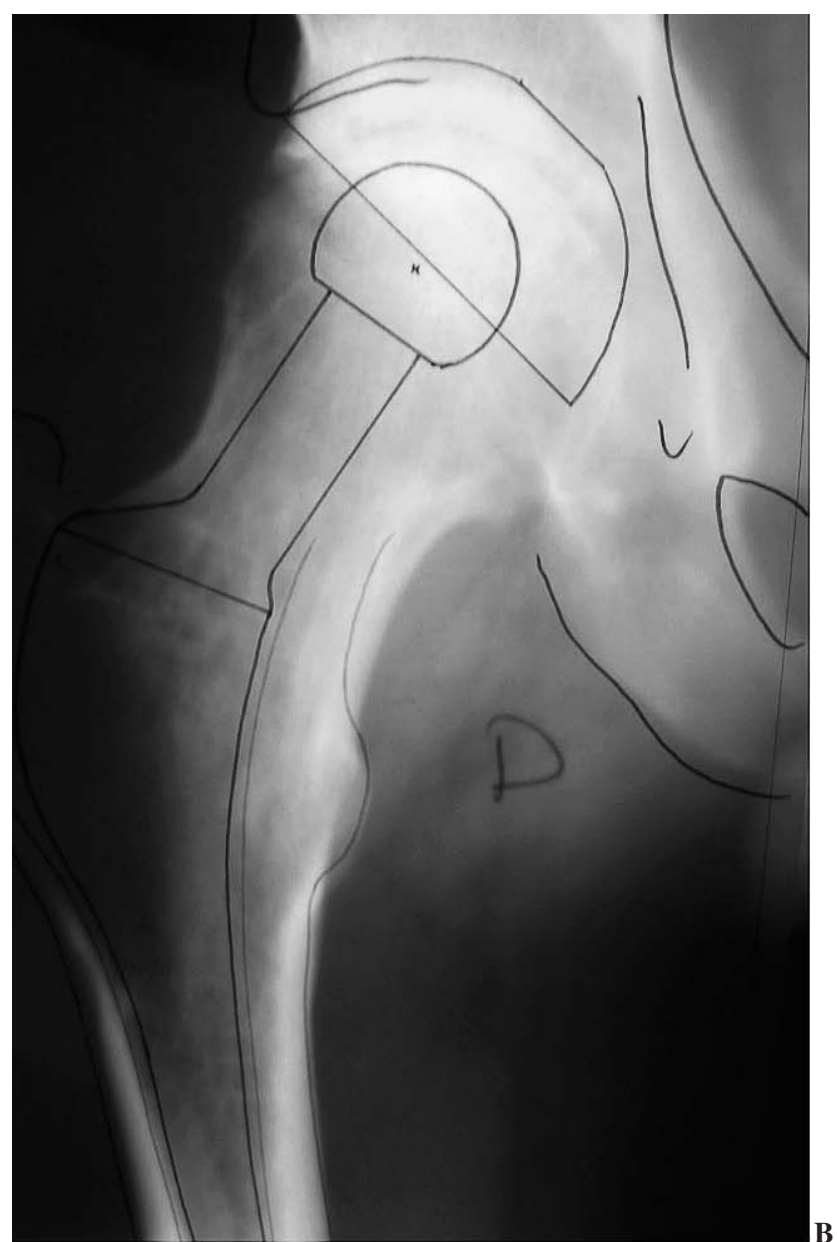

Figura 2. Planificación preoperatoria (A, B y C).

de Dall-Miles o material de osteosíntesis en caso de fractura; d) instrumental específico para extraer implante; cortar cerámica, etc.

\section{Medidas intraoperatorias}

Se debe procurar colocar los componentes en la posición óptima. Para ello se deben usar siempre los componentes de prueba para comprobar con ellos el arco de movimiento. 
Murcia A et al. Luxación recidivante de prótesis total de cadera
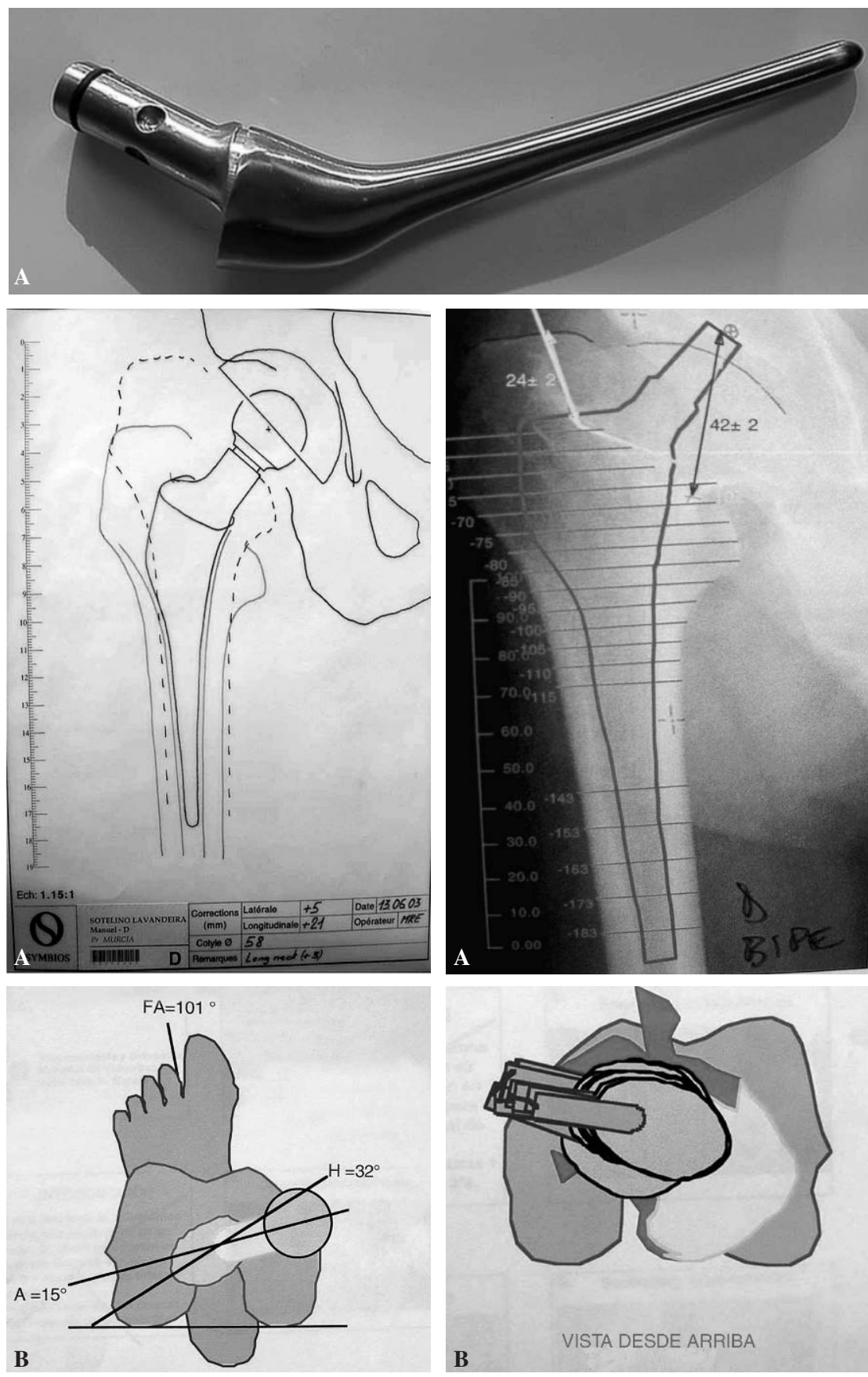

Figura 3. Planificación preoperatoria. (A) Prótesis total de cadera (PTC) a medida; (B) PTC a medida, tomografía axial computarizada y helitorsión.
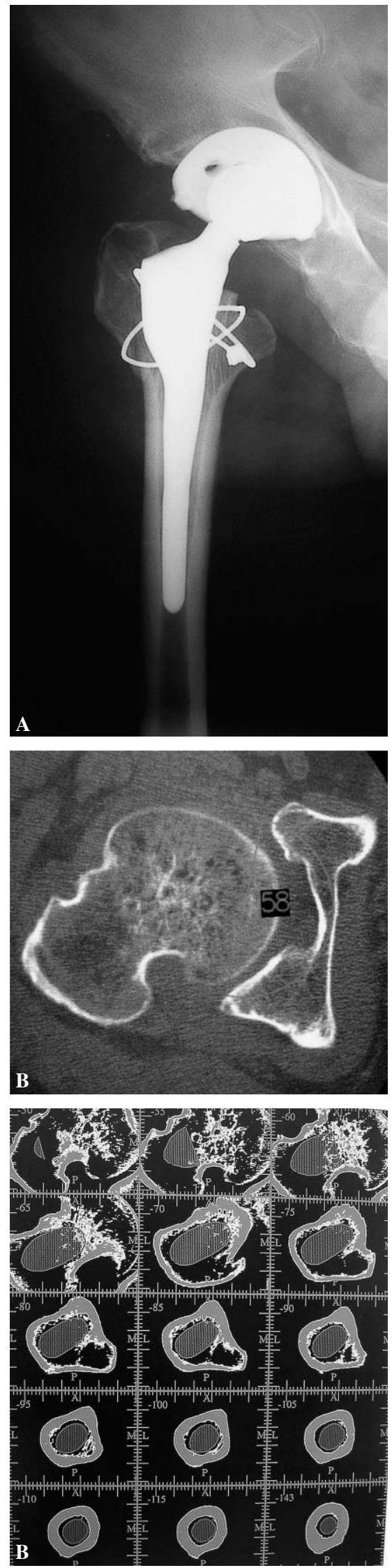
Del mismo modo se debe comprobar la adecuada tensión de las partes blandas, utilizando si hace falta vástagos con lateralización extendida, y además tener en cuenta la calidad de la anestesia, pues no es infrecuente tener dificultades para reducir los componentes de prueba debido a la excesiva tensión o contractura muscular que puede modificarse en el transcurso de la intervención.

Siempre que sea posible se debe evitar el uso de cabezas con faldas, ya que pueden producir choque contra los componentes acetabulares.

Utilizar juiciosamente los insertos con reborde y, por supuesto, colocarlos en el sitio adecuado dependiendo de la vía de acceso a la articulación.

Una de las mejores medidas para prevenir la luxación, que está documentalmente demostrada, es la correcta reparación y cierre de los tejidos seccionados para acceder a la articulación.

En el abordaje posterior cerrar con puntos transóseos la cápsula y los rotadores externos ${ }^{13-15}$. Si se ha realizado una vía de acceso anterior se debe reconstruir rigurosamente la musculatura abductora (fig. 4). Pero, la mejor recomendación durante la realización de la cirugía es «no salir del qui- rófano con la más mínima duda sobre la estabilidad de la artroplastia que acabamos de realizar» ${ }^{16}$.

\section{Cuidados postoperatorios}

Es fundamental la educación del paciente en las medidas que debe observar durante el postoperatorio inmediato. Se debe aconsejar no utilizar asientos bajos, así como un suplemento para el inodoro, tener especial cuidado al entrar o salir de un automóvil y adoptar una posición adecuada al dormir (no dar vueltas en la cama). La mayoría de los centros entregan a los pacientes que van a ser intervenidos unas instrucciones escritas de las medidas que deben observar, tanto para el correcto funcionamiento de su implante como para mejorar su duración y evitar complicaciones $^{17}$.

Una buena medida, sobre todo en casos de cirugía de revisión, es la limitación de la movilidad utilizando las férulas ortopédicas, de las que a tal efecto se dispone, y en las que se puede graduar la limitación de la movilidad mediante la bisagra o rótula graduada que llevan incorporada. Pasadas las primeras semanas se puede ir aumentando el
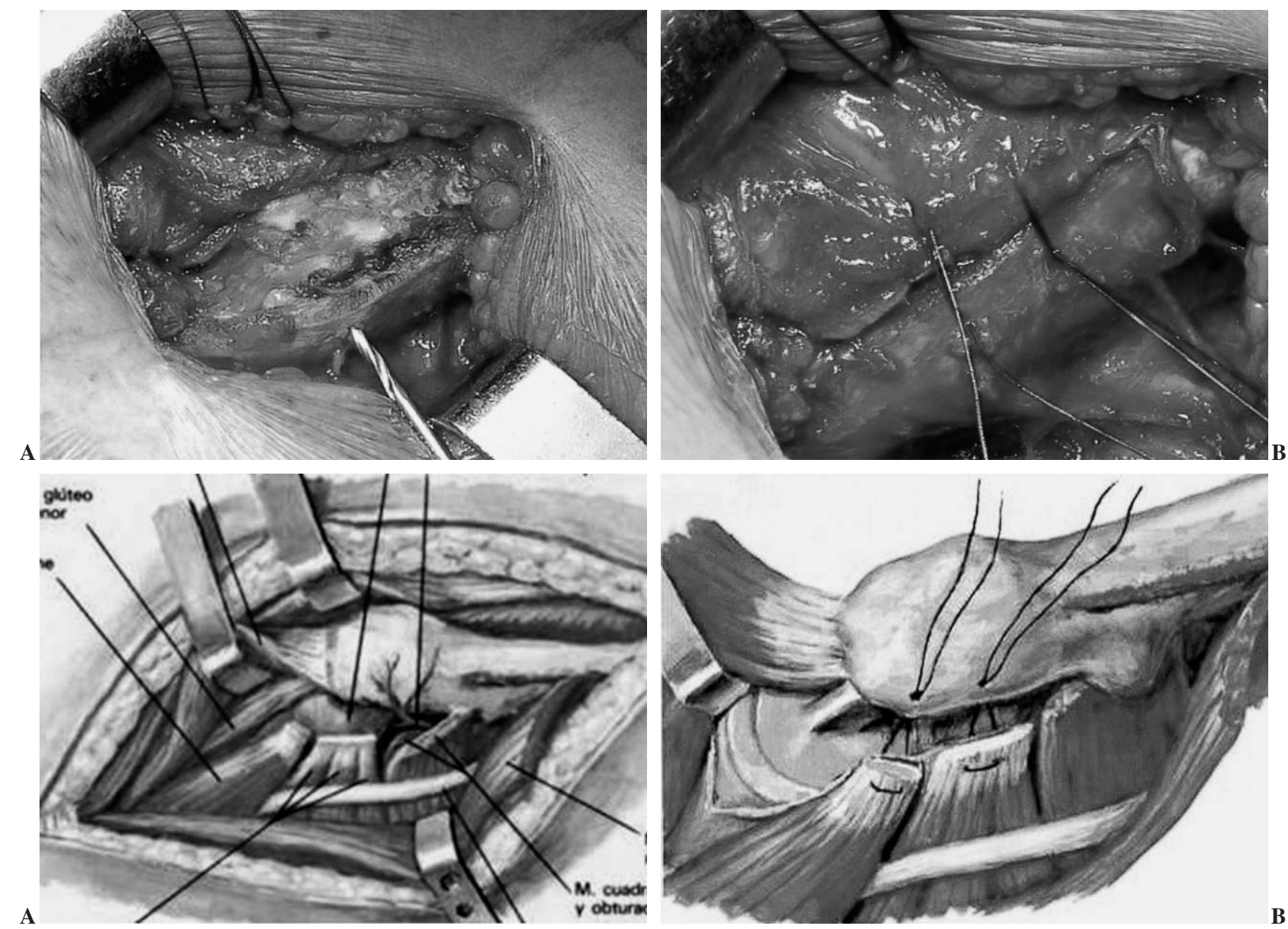

Figura 4. (A) Reconstrucción de musculatura abductora. Vía anterior. (B) reconstrucción de rotadores. Vía posterior. 

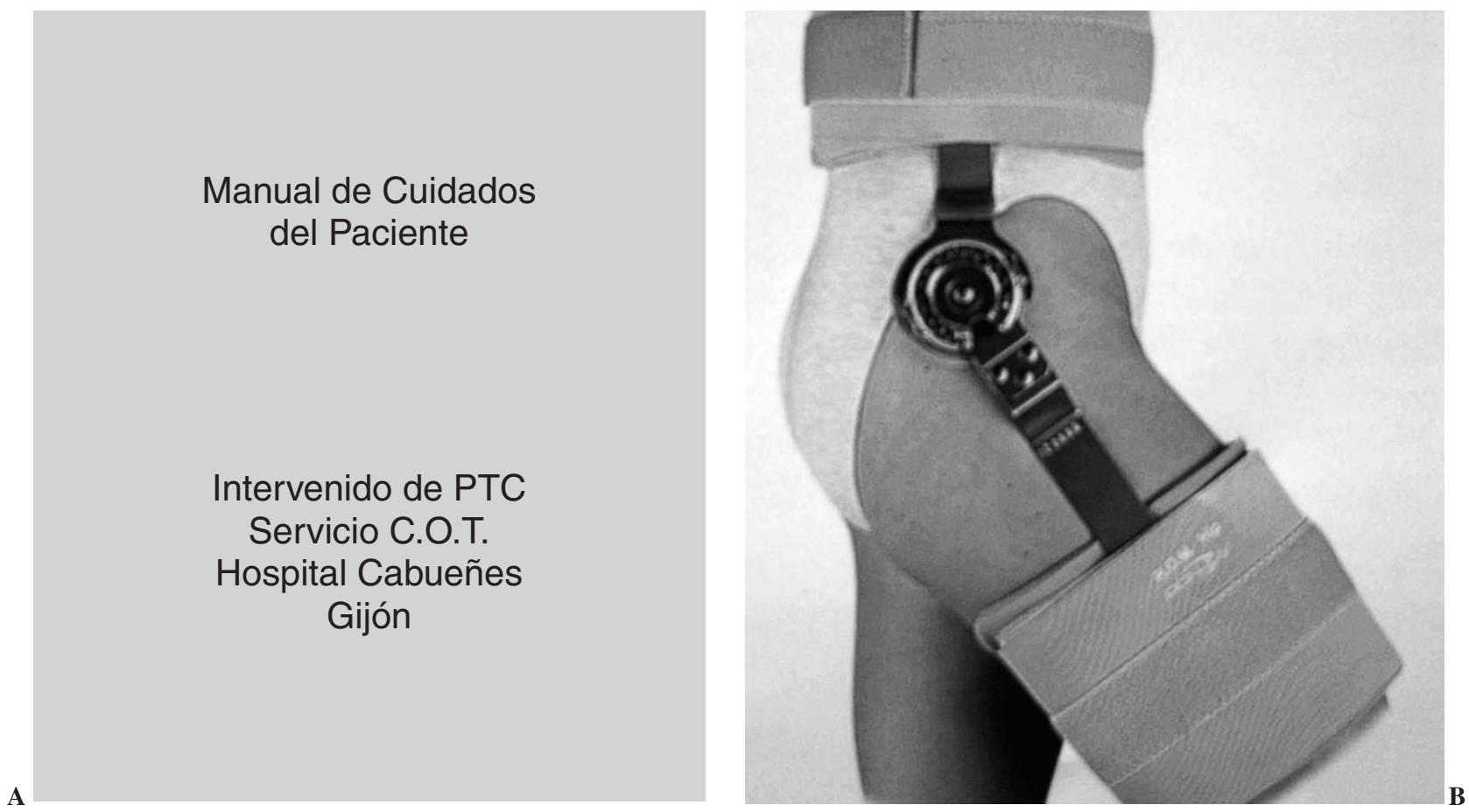

Figura 5. (A) Manual con recomendaciones. (B) Férula limitadora de movilidad.

arco de movimiento permitido, así como ser más permisivo en las restricciones anteriores (fig. 5).

\section{INFORMACIÓN NECESARIA PARA TRATAR LA LUXACIÓN}

Interesa preguntar qué movimiento se estaba realizando cuando se produjo la luxación; en ocasiones sucede al levantarse de una silla, de un asiento bajo o incluso el bidé; en otras al entrar o salir del baño, automóvil, etc. Lo más frecuente es que la cabeza protésica se salga hacia atrás, a través de un ojal en la cápsula posterior, y unas veces se desplaza hacia arriba, o menos frecuentemente hacia abajo. Es muy útil poder realizar la Rx de confirmación en proyección lateral (fig. 6), porque de esta forma se obtienen datos importantes para la toma de decisiones quirúrgicas. No se suele comprobar la estabilidad de la reducción, que daría datos concluyentes en la decisión quirúrgica.

Conviene recordar que una radiografía en proyección lateral verdadera con la cadera luxada ayuda muchísimo, tanto para establecer la dirección de la luxación como las medidas necesarias para su correcto tratamiento.

\section{TRATAMIENTO ORTOPÉDICO CERRADO}

La mayoría de las publicaciones reconocen que alrededor del $75 \%$ de las artroplastias luxadas se pueden tratar conservadoramente. Una vez diagnosticada la luxación se debe reducir bajo anestesia general y anotar la facilidad o dificultad de su reducción. Teóricamente sería muy útil poder comprobar la estabilidad de la reducción intentando reproducir la luxación pero, al igual que la crepitación de los fragmentos en el diagnóstico de una fractura, no suele hacerse.

Las medidas postreducción van dirigidas a evitar el movimiento responsable de la luxación y a prevenir un nuevo episodio durante el período de cicatrización de la brecha capsular; para ello algunos autores ${ }^{18-20}$ prefieren la colocación de una espica de yeso por un espacio de 6 semanas, o la colocación de las férulas limitadoras de la movilidad durante $12 \mathrm{se}-$ manas. Cada una de las propuestas especifican el porcentaje de éxito con el tratamiento propuesto. Estas pautas postreducción se utilizan preferentemente en Estados Unidos.

En nuestro medio se utiliza con más frecuencia un botín de yeso antirrotatorio por espacio de tres semanas, y posteriormente se continúa con la férula limitadora de la movilidad. El período de inmovilización tras el episodio de luxación depende de la sensación percibida por el cirujano durante la reducción de la estabilidad en que ha podido quedar la artroplastia. Por ello, en el período postreducción se aconseja la prescripción de una férula con movilidad controlada y limitada (fig. 5), descarga inicial y carga progresiva junto con un programa pausado de fisioterapia para obtener un buen control muscular ${ }^{5,7,20-22}$.

La mayor parte de las luxaciones se producen dentro de las 4-5 semanas del postoperatorio, y una vez reducidas se 


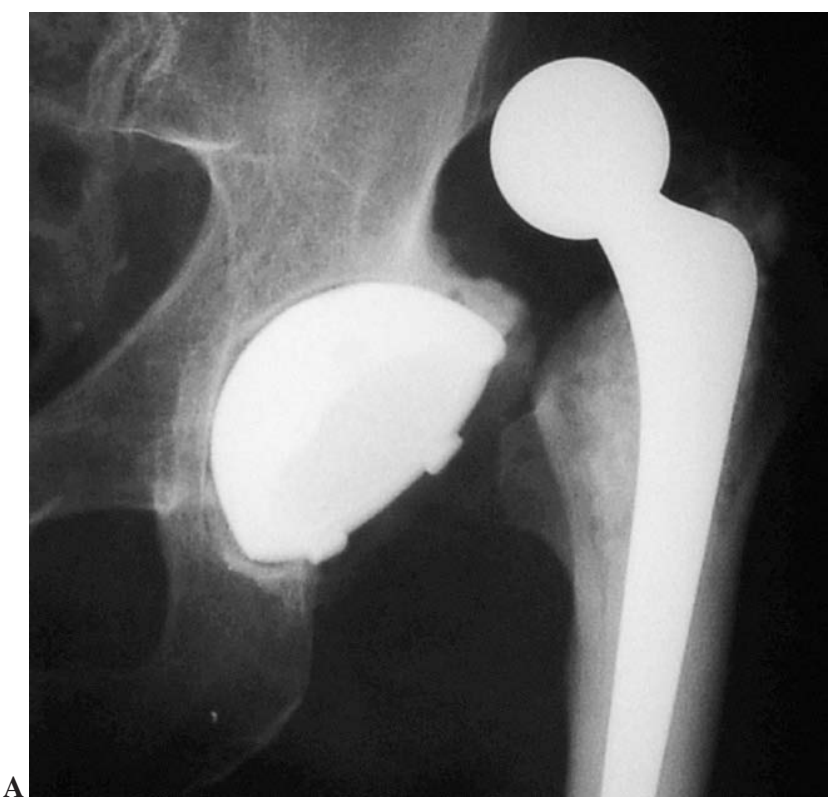

suelen estabilizar, por lo que se justifica una actitud expectante hasta el tercer-cuarto episodio.

\section{TRATAMIENTO QUIRÚRGICO}

El tratamiento quirúrgico de la luxación no es frecuente y se suele necesitar en uno de cada 100 pacientes, coincidiendo con que, históricamente, alrededor del $75 \%$ de las caderas luxadas se estabilizan mediante la cirugía ${ }^{23,24}$.

El mejor conocimiento de la inestabilidad y las causas que la originan, junto con el avance tecnológico ${ }^{8}$, hace que los resultados que se obtienen hoy día con el tratamiento quirúrgico de la inestabilidad sean mejores. El momento de presentación de la luxación también interviene en los resultados, ya que las que ocurren durante el período precoz, es decir, durante las 4-5 semanas, tienen menos posibilidades de convertirse en recidivantes que las que aparecen tardíamente. En general se admite que el tratamiento quirúrgico debe tenerse en cuenta a partir del tercer o cuarto episodio.

\section{Posibilidades de tratamiento quirúrgico}

Establecido en 1992 «el resultado del tratamiento quirúrgico de la inestabilidad de la artroplastia luxada, es mucho mejor si se conoce con precisión la causa de la inestabilidad y se realiza el tratamiento apropiado para corregirla ${ }^{23}$. Así se debe estudiar en cada paciente en particular los factores responsables de la inestabilidad, lo que quiere decir que la etiología de la inestabilidad determina el tratamiento, por lo que los factores que contribuyen a la inestabilidad pueden ser:

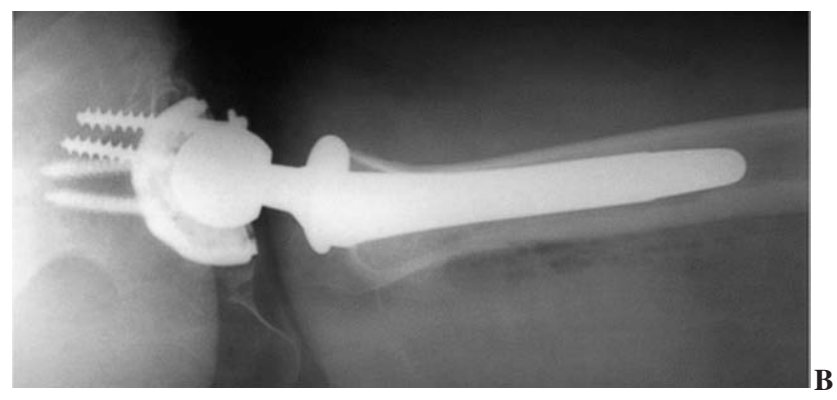

Figura 6. Imágenes de una luxación protésica de cadera. (A) Radiografía anteroposterior; (B) Radiografía lateral verdadera.

1. Malposición de los componentes: anteversión acetabular, que se aprecia en la Rx lateral verdadera; inclinación acetabular; anteversión del componente femoral.

2. Tensión inadecuada de las partes blandas.

3. Pinzamiento o impingement (óseo, partes blandas y prótesis).

4. Insuficiencia de la musculatura abductora.

5. Factores relacionados con el propio paciente (problemas neuromusculares, demencia, etc.).

\section{TRATAMIENTO DE LA MALPOSICIÓN DE LOS COMPONENTES}

Aunque puede ser la causa de cualquier tipo de luxación, independientemente del momento de su presentación, suele darse en las luxaciones tardías y se dispone de dos posibilidades: reorientar el componente malposicionado y utilizar cejas elevadoras.

\section{Reorientar el componente malposicionado}

Habitualmente es el componente acetabular el que está malorientado y debe ser tratado, ya que la orientación del componente femoral es fácilmente detectable si se evalúa su plano de anteversión en relación con el plano de flexión de la rodilla. Se reconoce ${ }^{23}$ un $80 \%$ de éxito cuando se reorienta el cotilo. Conviene recordar que a veces la vía de acceso a la articulación influye desfavorablemente y contribuye a ser la responsable de la malorientación. En el acceso posterior, al estar borrada la lordosis lumbar, si el cirujano está acostumbrado a la vía anterior, se suele colocar en menos anteversión de la necesaria, ya que cuando el paciente se 
pone de pie y reaparece la lordosis lumbar, el componente acetabular tendrá la anteversión de la cirugía menos la que resta la lordosis lumbar restablecida.

\section{Cejas elevadoras}

El componente acetabular bien fijado en anteversión o retroversión excesivas se puede hacer más continente sin necesidad de recambiarlo, mediante el atornillado de un sector, obtenido por corte, de una porción de otro cotilo de polietileno ${ }^{25}$, convirtiéndolo en un cotilo con reborde. Sin embargo, esta solución artesanal no ha significado una mejoría en los resultados obtenidos, y son frecuentes los fracasos. Así lo corrobora nuestra experiencia personal, que aunque se limita a tres casos, fracasó en todos ellos.

Actualmente, la modularidad de los componentes permite respetar la parte metálica fija del acetábulo y recambiar el inserto de polietileno por uno con ceja o reborde apropiados. En cualquier caso, también puede ocurrir que debido al desgaste, o por las maniobras en la extracción o recambio, el mecanismo de seguridad del inserto se haya deteriorado, en cuyo caso hay que recurrir a cementar, haciendo las pertinentes rugosidades en la superficie externa del cotilo de polietileno, un nuevo inserto al que hay que dar la anteversión adecuada.

Se debe tener en cuenta que el procedimiento artesanal de reorientar el cotilo añadiéndole un reborde atornillado no aumenta la zona de seguridad de la cadera. Así, atornillando un reborde posteriormente mejora o aumenta la estabilidad posterior, pero disminuye la anterior y viceversa. Además, la cuantía de reorientación que proporciona un reborde elevado es moderada, y en los casos de malposición franca es necesario el recambio completo del componente acetabular.

\section{TRATAMIENTO DE LA INADECUADA TENSIÓN DE PARTES BLANDAS}

El restablecimiento de la tensión de las partes blandas es fundamental para mantener la estabilidad de la cadera. La avulsión y ascenso del trocánter mayor osteotomizado supone un riesgo, por lo que se ha aconsejado la reinserción profiláctica del mismo, descendiéndolo y adelantándolo para limitar la rotación interna. Sin embargo ${ }^{22}$ el $80 \%$ de las prótesis luxadas, que presentaban ausencia de unión del trocánter, permanecieron estables después de la reducción cerrada inicial, por lo que la reinserción del mismo parece aconsejable sólo si se producen recidivas (fig. 7).

Ekelund ${ }^{26}$ publica que el descenso del trocánter mayor, esté o no bien fusionado, y su reinserción más distal con el miembro en abducción han mostrado resultados satisfactorios en la estabilización de la prótesis, sin defectos de orientación en 19 de 21 pacientes. En el estudio se incluyen 7 casos de ATC tras osteosíntesis fracasada en fracturas subcapitales, que se sabe que constituyen un grupo de alto

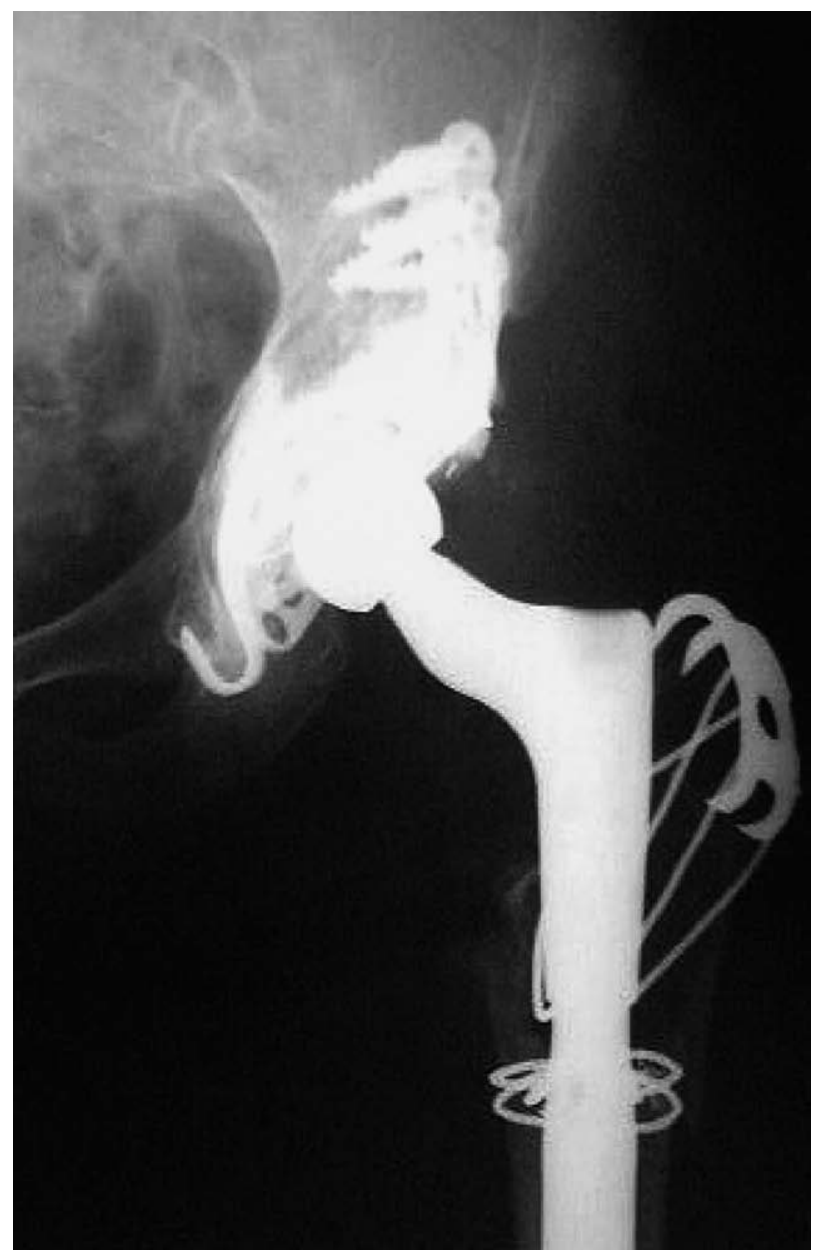

Figura 7. Radiografía con anillo antiprotrusión. Vástago modular con distintos offset.

riesgo de inestabilidad. Se recomienda igualmente sistemas modernos de fijación como las grapas con cables de DallMiles, dada la frecuentemente escasa consistencia de la estructura ósea en esta zona.

La tensión de las partes blandas se puede conseguir mediante:

1. El aumento de la longitud del cuello.

2. La revisión del componente femoral y colocar otro con más cuello.

3. La utilización de insertos acetabulares excéntricos.

4. La tensión y trasposición distal del trocánter mayor.

\section{TRATAMIENTO DEL IMPINGEMENT}

Conviene recordar que exiten tres tipos de impingement:

1. Óseo. Pelvis-trocánter mayor-osificación heterotópica.

2. Producido por las partes blandas.

3. Producido por la prótesis.

Una relación pequeña diámetro cabeza-cuello aumenta las posibilidades de impingement. 
La combinación de un cuello modular largo con faldón y un elevado reborde acetabular puede ser causa de un impingement precoz.

El tratamiento consiste en eliminar la fuente del impingement dependiendo de la etiología y tipo del mismo ${ }^{24}$.

La proporción de éxitos depende de la eficacia del tratamiento, pero existen pocos datos en la literatura. En la serie más citada de Daly y Morrey se cifra alrededor del $50 \%{ }^{23}$.

\section{TRATAMIENTO COMBINADO CUANDO EL PROBLEMA ES MULTIFACTORIAL}

Para la mayoría de los pacientes con inestabilidad de cadera, como la causa es multifactorial, es necesario un tratamiento combinado que conduzca a la estabilidad:

1. Optimizar la posición de los componentes.

2. Optimizar la tensión de las partes blandas, aumentando la longitud del cuello y descender y tensar el trocánter mayor.

3. Eliminar la causa o causas del impingement.

4. Optimizar la relación diámetro cabeza-cuello, que suele conseguirse aumentando el diámetro de la cabeza protésica.

\section{INSERTOS MODULARES Y RECAMBIO DE LA CABEZA AISLADA}

El recambio aislado del inserto y cabeza tienen una tasa de éxito entre el 55 y el 77\%, dependiendo de las series publicadas $^{27,28}$. La mayoría de las técnicas y tratamientos propuestos para conseguir la estabilidad de la cadera son mucho más eficaces cuando se puede identificar la causa de la inestabilidad y resolverla adecuadamente, pero con relativa frecuencia no siempre se puede diagnosticar la causa exacta de la inestabilidad, y por consiguiente resolverla con éxito por medio de procedimientos estándar.

\section{EN CASOS SELECCIONADOS CONVERTIR EL ACETÁBULO EN BIPOLAR O COTILO CONSTREÑIDO}

Dentro de este apartado se pueden considerar diversas posibilidades:

\section{Convertir la artroplastia en bipolar}

El convertir una artroplastia convencional en una bipolar puede recuperar la estabilidad de la articulación. Se publican cifras de éxito entre el 80 y el $100 \%$. A favor de esta solución hay constataciones de su eficacia, como han demostrado Zelicof y $\operatorname{Scott}^{29}$, pues sobre 11 casos de caderas inestables no han tenido ninguna recidiva.
Otras series ${ }^{30}$ obtienen el $100 \%$ de éxito en tres casos de caderas inestables ampliando el tamaño de la cabeza y transformando la artroplastia en bipolar. Está demostrado que la naturaleza de la biarticular o bipolar junto con el aumento del diámetro externo de la cabeza mejoran la estabilidad.

En contra también existen circunstancias, como la permanencia de algunas artroplastias dolorosas, y la erosión del acetábulo junto con la migración, que son altas en las artroplastias bipolares cuando se utilizan en casos de cirugía de revisión, sobre todo en casos de pérdida de masa ósea acetabular ${ }^{31}$.

Los modelos «tripolares» no están sujetos a estos problemas, pero precisan insertos a medida.

\section{Conversión a cotilo constreñido o con mecanismo de bloqueo}

Estos implantes que capturan la cabeza femoral proporcionan una estabilidad que permite resolver muchos de los problemas que hasta ahora eran intratables. Hay una gran variabilidad en el diseño de estos implantes constreñidos, que a la vez proporcionan distintos grados de constricción, y que además están sujetos a distintas formas de fallo. En general hay tres tipos principales de implantes constreñidos: cotilos que reciben en su interior más de la mitad de la cabeza (ecuador) y que emiten un chasquido cuando la capturan, otros en que la constricción la proporciona un anillo metálico alrededor de la periferia de un inserto de polietileno y los implantes constreñidos tripolares.

Los primeros se caracterizan además porque la cabeza protésica se encuentra más penetrada de lo normal y por consiguiente, al no disponer de ningún mecanismo de estrangulación, son los que determinan menor constricción (figs. 8, 9 y 10).

Los diseños con mecanismo de bloqueo pueden fallar por desencaje de dicho mecanismo, por luxación de la cabeza sobre el inserto, o por fallo a nivel de cotilo-hueso, hueso-cemento, implante-cemento o entre el inserto de polietileno y el soporte metálico.

\section{A favor}

Se produce una estabilidad inmediata; es una técnica sencilla, que incluso permite mantener el cotilo metálico en su posición, sobre todo si está firme, y cementar un inserto constreñido.

Las publicaciones refieren porcentajes entre el $67 \mathrm{y}$ el $96 \%$ de resultados favorables.

\section{Resultados}

Goetz et $\mathrm{al}^{32}$ reportan 53 caderas (tripular constreñido) con más del $90 \%$ de buenos resultados a dos años.

Anderson, Murray y Skinner ${ }^{33}$ comunican 18 caderas (cotilo constreñido con mecanismo de bloqueo) con un $67 \%$ de resultados favorables. 
A
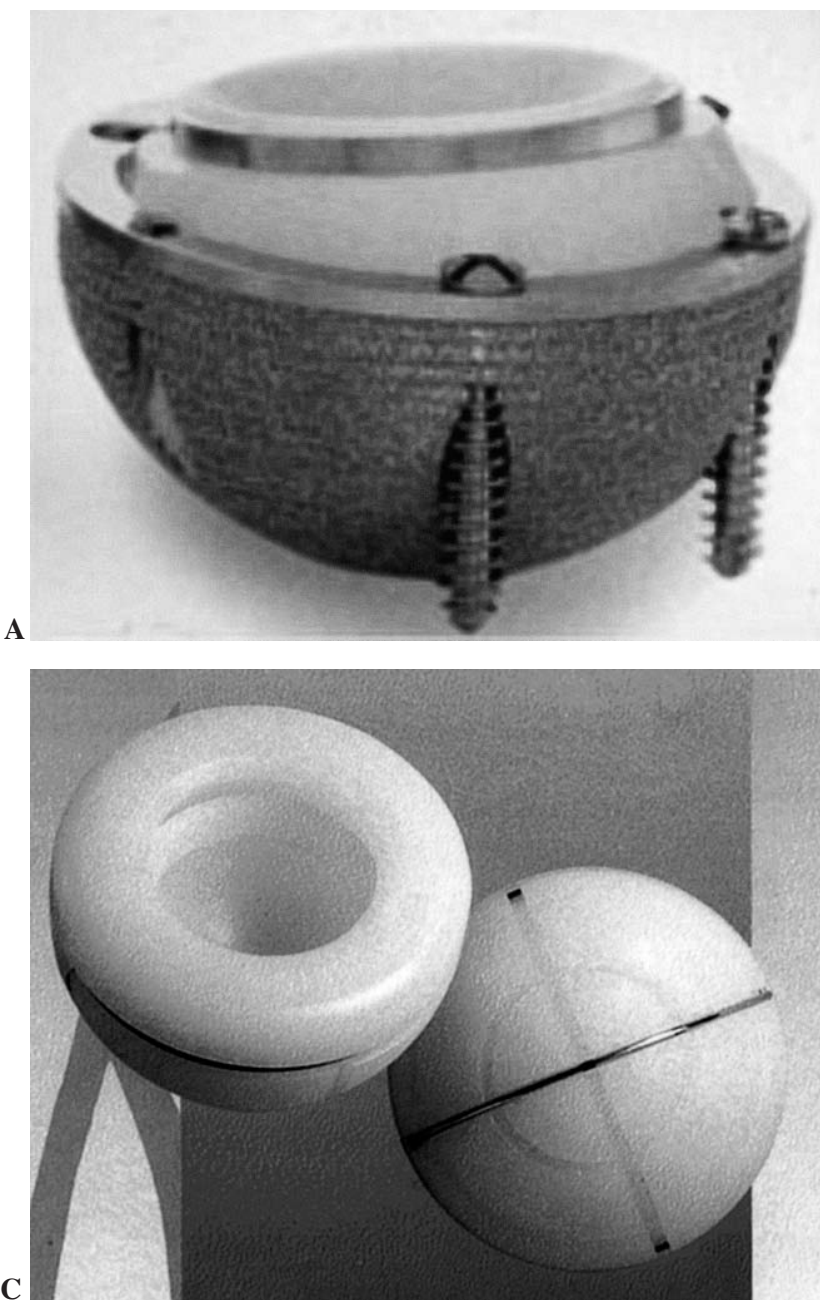

Figura 8. Diversos cotilos constreñidos $(A, B, C)$ y radiografía de cotilo constreñido $(D)$

Los fracasos posibles se pueden presentar en cualquiera de las tres intercaras: a) inserto-cotilo metálico; b) cabezainserto y c) mecanismo de bloqueo.

\section{En contra}

No todos los cotilos constreñidos proporcionan igual cantidad de constricción.

El rango de movilidad de la mayoría de los cotilos constreñidos, antes del choque entre el cuello y el inserto, es pequeño.
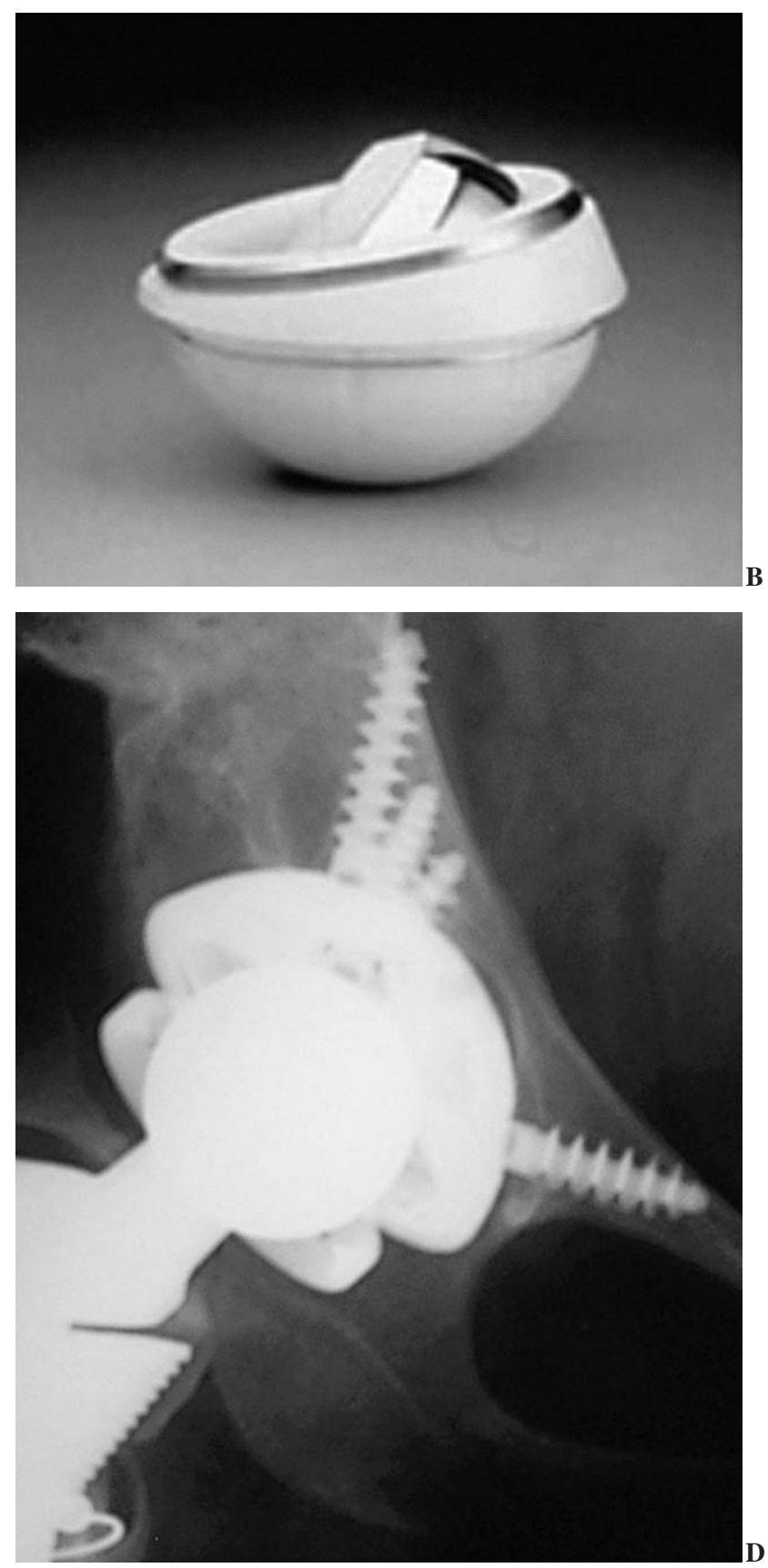

Se produce un aumento del desgaste del polietileno.

Una complicación no frecuente es la disociación conocabeza, siendo necesaria la reducción abierta y aumentar la longitud del cuello con o sin faldón (fig. 11).

Existe la posibilidad de fracaso en las dos intercaras entre el cotilo metálico y el hueso y entre el cotilo metálico y el inserto debido a la transmisión de cargas excesivas a estas dos intercaras.

Habitualmente, si la cadera se vuelve a luxar, al quedar la cabeza aprisionada y constreñida o fuera del inserto, es necesaria la cirugía abierta, aunque tenemos dos casos en 
Murcia A et al. Luxación recidivante de prótesis total de cadera
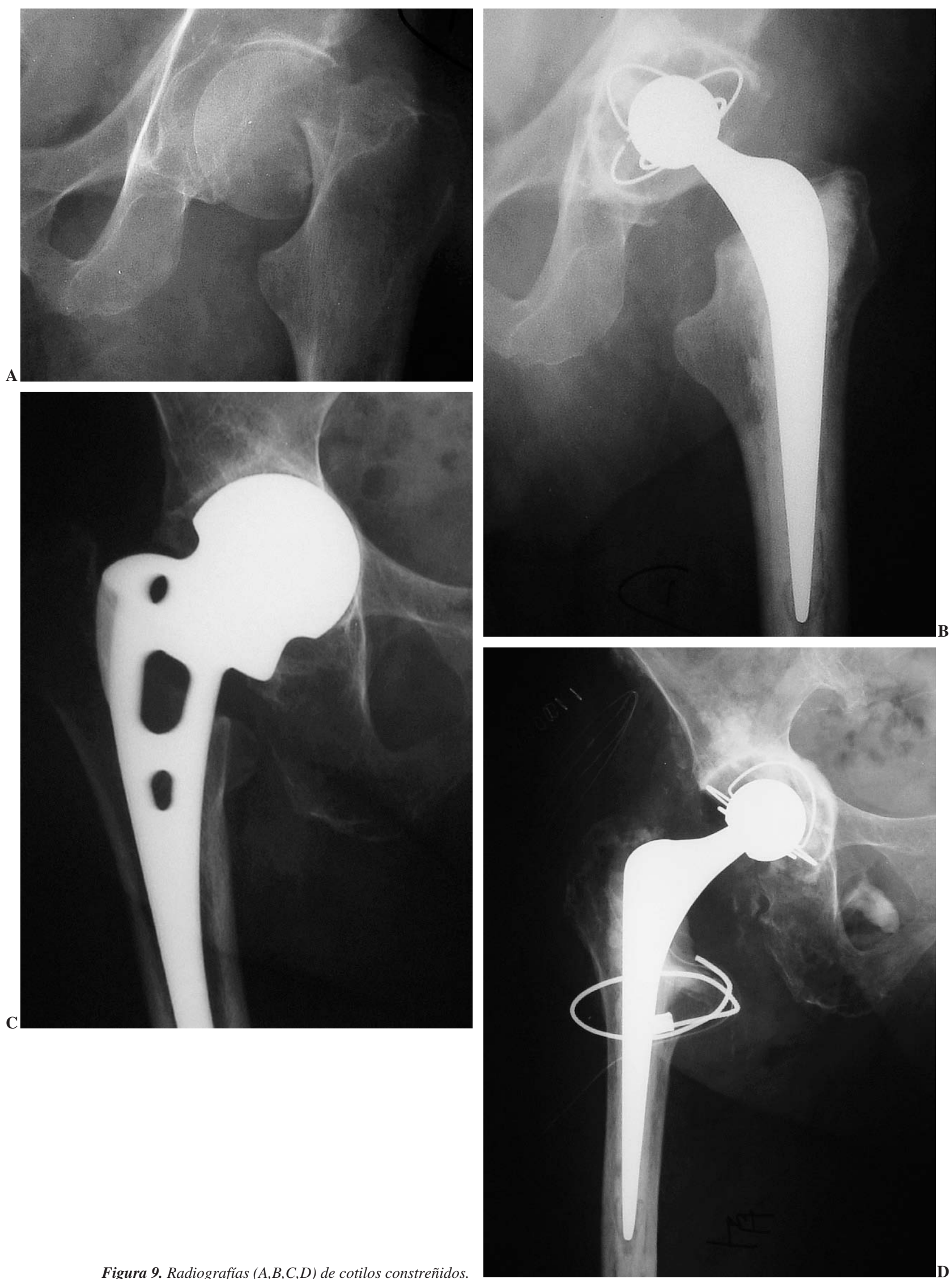

Figura 9. Radiografías $(A, B, C, D)$ de cotilos constreñidos. 

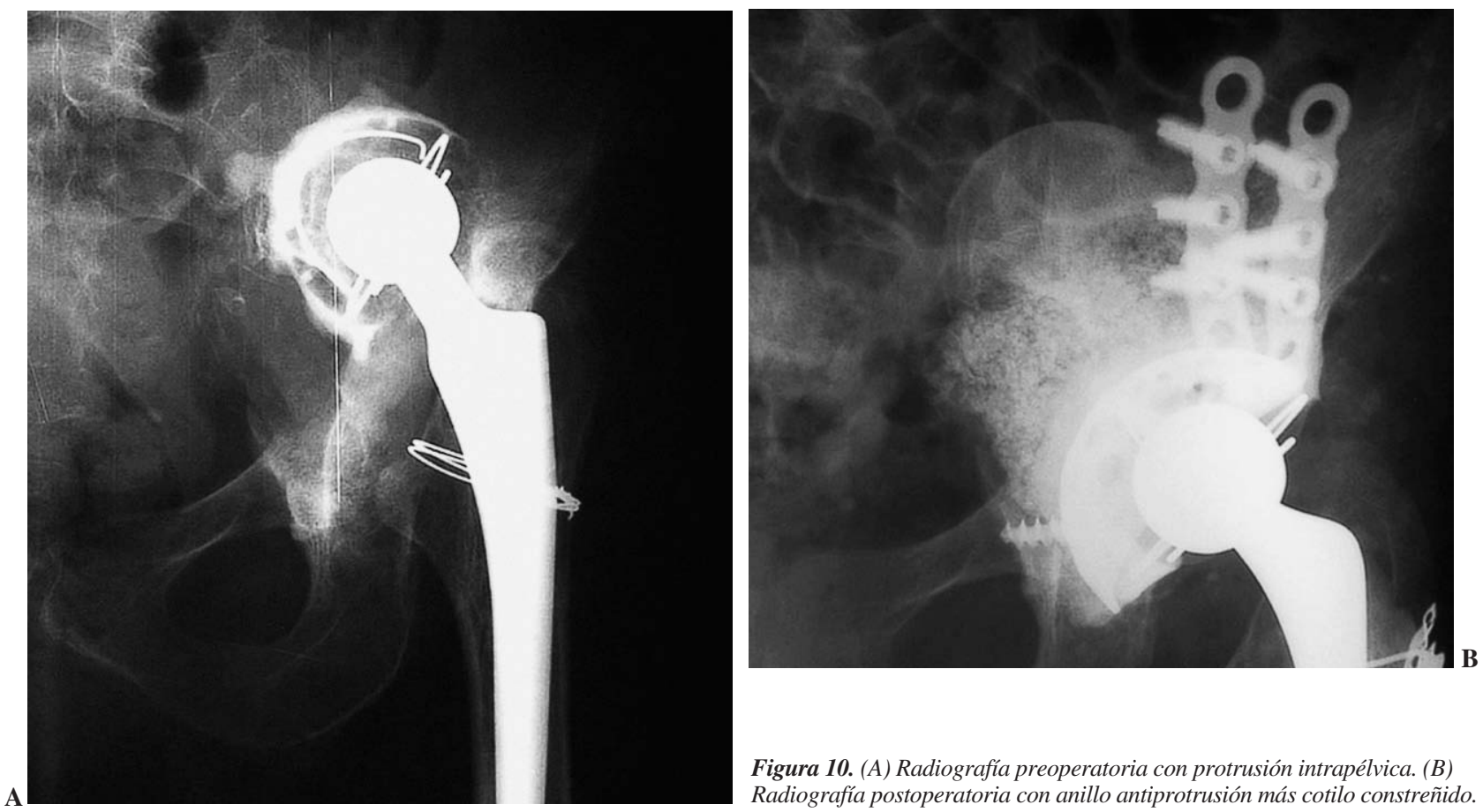

Figura 10. (A) Radiografía preoperatoria con protrusión intrapélvica. (B) Radiografía postoperatoria con anillo antiprotrusión más cotilo constreñido.
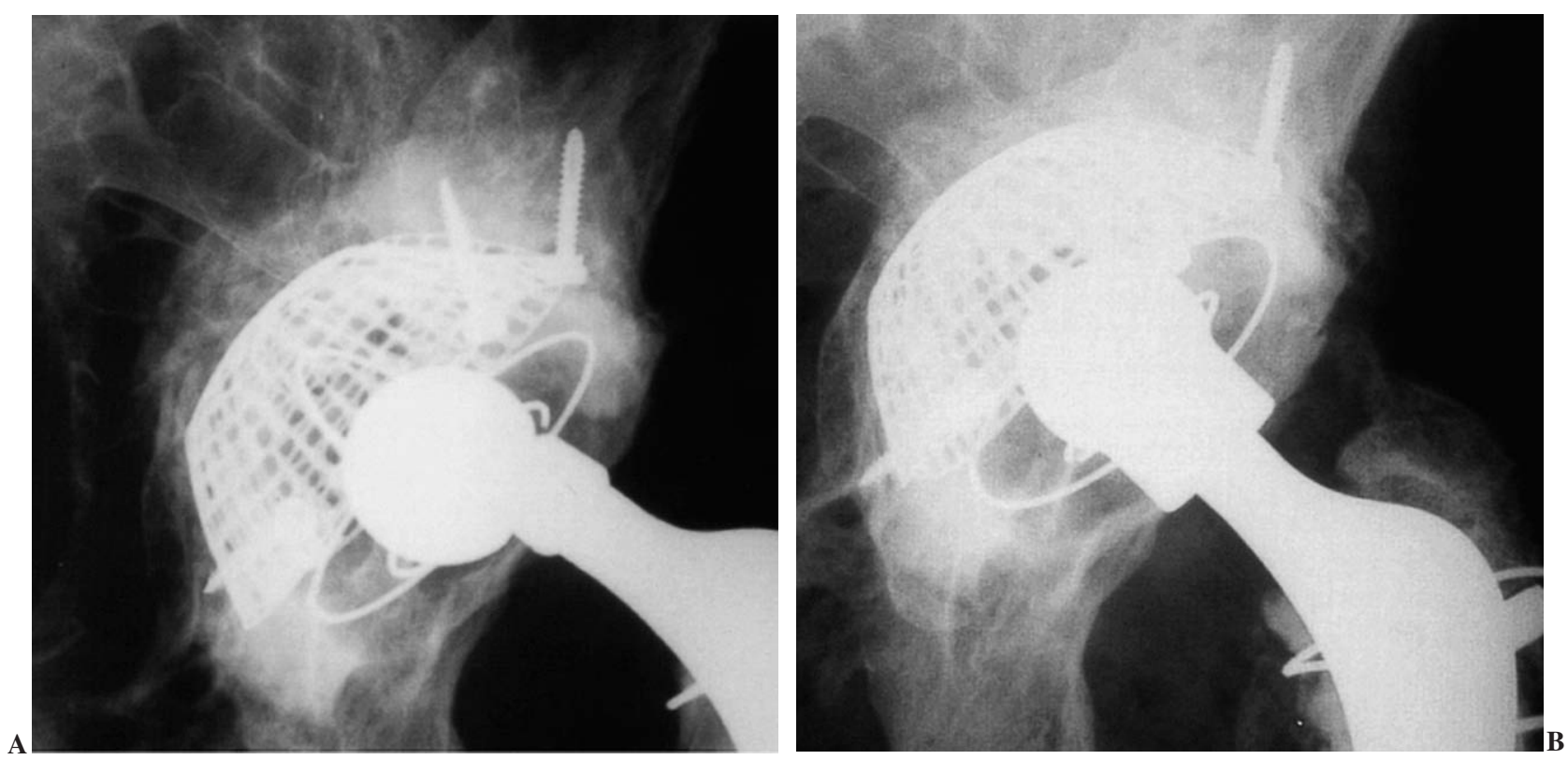

Figura 11. (A) Disociación cono-cabeza. (B) Radiografía de cabeza cuello más largo y con faldón.

los que se ha podido evitar la cirugía, siendo favorable la reducción cerrada.

\section{Indicaciones relativas a los insertos constreñidos}

1. Los fracasos de: a) intentar estabilizar la cadera mejorando la posición de los componentes; b) tensión adecuada de las partes blandas; c) quitar las posibles causas de impingement.
2. Pacientes con deficiencia importante del mecanismo abductor

3. Pacientes incapaces de colaborar con las medidas mínimas de prevención de luxación.

4. Pacientes mayores en que los componentes están razonablemente bien colocados y con buen aspecto de osteointegración, siempre y cuando quepa el inserto constreñido dentro del cotilo metálico. 


\section{Detalles técnicos}

En primer lugar se deben conocer las características y particularidades del cotilo constreñido que uno quiere utilizar. A veces, introducir la cabeza o el mecanismo de bloqueo precisa de algún instrumento especial.

Es importante comprender y saber qué cantidad de constricción aporta cada modelo en concreto.

Si se cambia el cotilo metálico hay que asegurar una estabilidad primaria importante, lo más rígida posible, con cuantos tornillos se puedan colocar, con el fin de reducir la posibilidad de un fracaso temprano por aflojamiento en la interfaz cotilo-hueso.

\section{Utilización de cabezas de mayor diámetro}

Cada día hay más partidarios de recurrir a cabezas de mayores diámetros, que dan más estabilidad y que hoy no plantean los antiguos problemas de las superficies de fricción.

Los resultados publicados son escasos, pero alentadores, con cifras del $100 \%{ }^{34}$.

En cualquier caso, tienen una serie de ventajas: mayor rango de movilidad previo a la luxación, disminuyen la posibilidad de impingement, mayor resistencia a la luxación, incluso en caso de que tenga lugar un impingement, puede mejorar la estabilidad capsular, si ocurriera una luxación es posible su reducción cerrada y es factible conseguirlas en todos los fabricantes.

Las desventajas son que las cabezas de gran diámetro permiten menor grosor de polietileno, aunque con los polietilenos hiperenlazados esta desventaja es menos importante, el impingement de tipo no protésico es posible y todavía no existe un buen soporte en la literatura ${ }^{21}$.

Aunque se ha repetido en distintas ocasiones, la prevención es el mejor tratamiento de las luxaciones; hoy día se dispone de mejores posibilidades para el tratamiento eficaz de la luxación. Antes se podía sustituir el componente cotiloideo por un sistema bipolar. Los componentes modulares permiten más opciones para el tratamiento. Igualmente, los polietilenos de enlaces entrecruzados son más resistentes y permiten, al ser más delgados, tener una supervivencia mayor. Se dispone también de insertos excéntricos y de insertos constreñidos que aumentan las posibilidades de estabilidad. Las cabezas protésicas de mayor diámetro disminuyen las posibilidades de nuevas luxaciones. Una posibilidad técnica que cada vez tiene más adeptos consiste en cementar un inserto más delgado, pudiendo dejar in situ un componente metálico y facilitar enormemente la cirugía $^{35,36}$.

Por último, no hay que olvidar que, aparte de la complicación que supone una luxación recidivante con las consiguientes intervenciones quirúrgicas para estabilizar la cadera, puede sumarse a ella la infección. La asociación de inestabilidad de cadera protésica e infección, desafortunada-

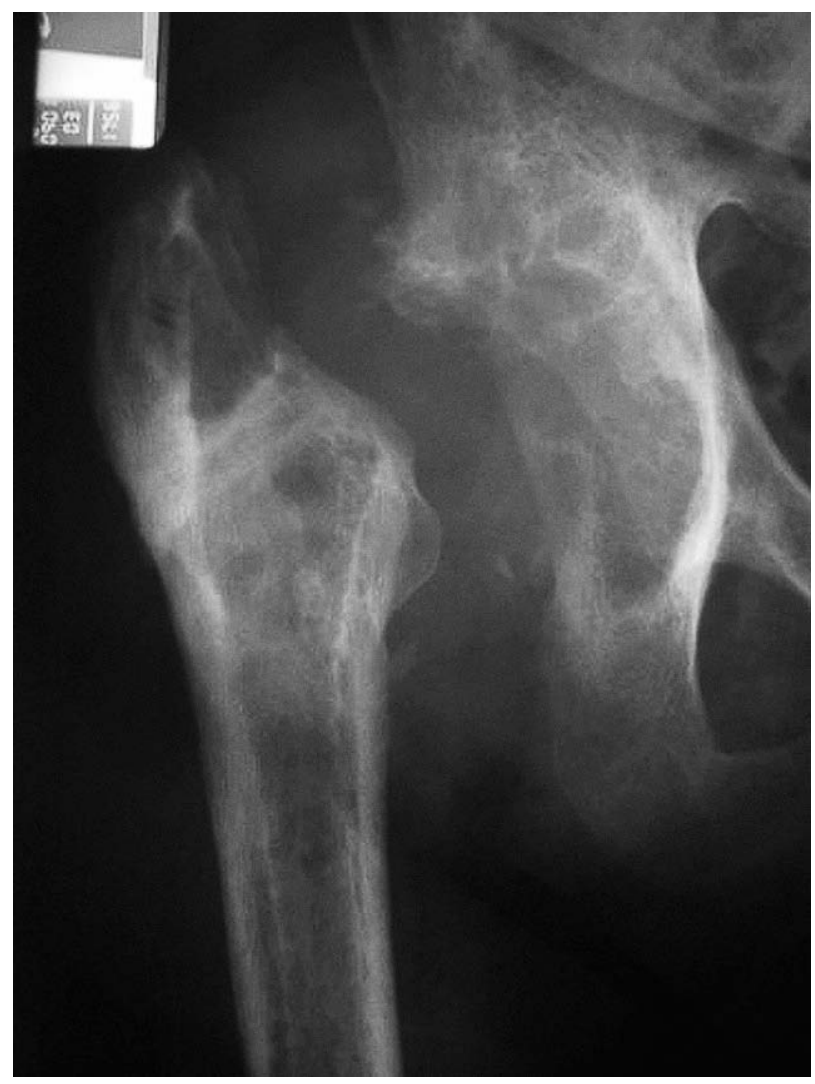

Figura 12. Artroplastia-resección de Girdlestone.

mente, en ocasiones, acaba en artroplastia-resección de Girdlestone, que probablemente sea de las pocas indicaciones de este tipo de cirugía hoy día (fig. 12).

\section{BIBLIOGRAFÍA}

1. Wroblweski MB. Long-term results of low friction arthroplasty. Curso de artroplastias. Universidad Autónoma de Barcelona; 2005.

2. Masonis JL, Bourne RB. Surgical approach, abductor function and total hip arthroplasty dislocation. Clin Orthop. 2002;405:46-53.

3. Katz JN, Losina E, Barrett J, Phillips CB, Mahomed NN, Lew RA, et al. Association between hospital and surgeon, procedure volume and outcomes of total hip replacement in the United States medicare population. J Bone Joint Surg Am. 2001;83A:1622-9.

4. Mahoney CR Pellici PM. Complicaciones de la artroplastia total de cadera primaria: cómo evitar y tratar las luxaciones. En: Orthopaedic Knowledge Update. Ed Koval. Edición en español. Extremidades inferiores; 2004. p. 39-49.

5. Munuera L. Luxación de la Prótesis Total de Cadera. En: Luxaciones. Curso Básico Fundación SECOT. Universidad de Oviedo; 2000.p.255-63.

6. Callaghan JJ. Recurrent dislocation: constrained sockets are the answer. In the affirmative. Current Concepts in Joint Replacement. Winter; 2002. 
7. García-Cimbrelo E, Munuera L. Dislocation in low friction arthroplasty. J. Arthroplasty. 1992;7:149-54.

8. Berry DJ. Unstable total hip arthroplasty: prevention and management. Hip. 2002;291-3.

9. Thornhill TS. Dislocation: Prevention is easier than the cure. Current Concepts in Joint Replacement. Winter; 2002.

10. Maestro A, Suárez MA, Martínez J, et al. La selección del implante en la artroplastia total de cadera. Planificación preoperatoria. En Artroplastias no cementadas de Cadera. Servicio de Publicaciones Universidad de Oviedo; 1997. p. 41-52.

11. Olmedo-García N, López-Prats F. Planificación preoperatoria de la artroplastia total de cadera no cementada. En: Patología de la cadera en el adulto. Curso Básico Fundación SECOT. Alicante; 2004. p. 175-81.

12. Sledge CB, Sledge JB. Planificación. En: Máster en Cirugía Ortopédica. Cadera. Madrid: Ed. Marban; 1999. p. 211-6.

13. Sculco TP. Abordaje posterior. En: Máster en Cirugía Ortopédica. Cadera. Madrid: Ed. Marban; 1999. p. 3-14.

14. Murcia-Mazón A. Influencia de la vía de abordaje en el resultado de la cirugía del aparato locomotor. En: Manejo pre, intra y postoperatorio del enfermo quirúrgico. Oviedo: Ed. Universidad de Oviedo. Servicio de Publicaciones; 1994. p. 283-91.

15. Dixon MC, Scout RD, Schai PA, Stamos V. A simple capsulorraphy in a posterior approach for total hip arthroplasty. J Arthroplasty. 2004;19:373-6.

16. Cabanela M. Dislocation after THA: Avoidance and management. The Hip Society. Thirty-Third Open Meeting of the Hip Society. AAOS Washington Course. 2005;362:8-12.

17. Deroche P. Hip Prostheses and information for patients. En: Arthroplastie Totale de Hanche. Quelle sera la prothèse du 21me siecle? Lyon: Ed. Agora Group; 2000. p. 351-9.

18. Ritter MA. Dislocation and subluxation of the total hip replacement. Clin Orthop. 1976;121:92.

19. Dorr LD, Wolf AW, Chandler RW, Conventry JP. Classification and treatment of dislocation of total hip arthroplasty. Clin Orthop. 1983;173:151-8.

20. Vosburgh CL, Vosburgh JB. Closed reduction of total hip arthroplasty dislocation. The Tulsa technique. J Arthroplasty. 1995;10:693-4.

21. Hedlundh U, Sanzén L, Fredin H. The prognosis and treatment of dislocated total hip arthroplasties with a $22 \mathrm{~mm}$ head. J. Bone Joint Surg Br. 1997;79B:374-8.

22. Joshil A, Lee CM, Marcowic L, et al. Prognosis of dislocation after total hip arthroplasty. J Arthroplasty. 1998;13:17-21.

23 Daly PJ, Morrey BF. Operative correction of the unstable total hip arthroplasty. J Bone Joint Surg Am. 1992;74A:1334-43.

24. Fraser GA, Wroblewski MB. Revision of the Charnley lowfriction arthroplasty for recurrent or irreducible dislocation. J Bone Joint Surg Br. 1981;63B:552-5.

25. Olerud S, Karlström G. Recurrent dislocation after total hip replacement. Treatment by fixing and additional sector to the acetabular component. J. Bone Joint Surg Br. 1985;67B:402-5.
26. Ekelund A. Trochanteric osteotomy for recurrent dislocation of total hip arthroplasty. J Arthroplasty. 1993;8:629-32.

27. Earll MD, Fehring TK, Griffin WL, Mason JB, McLay T, Odum S. Success rate of modular component exchange for the treatment of an unstable total hip arthroplasty. J Arthroplasty. 2002;17:864-9.

28. Toomey SD, Hopper RH, McAuley JP, Engh CA. Modular component exchange for treatment of recurrent dislocation of a total hip replacement in selected patients. J Bone Joint Surg Am. 2001;83A:1529-33.

29. Zelicof SB, Scott RD. Abstract: Conversion to bipolar arthroplasty for the treatment of recurrent total hip dislocations: A two-to-seven-year follow-up study. 59 ${ }^{\text {th }}$ Annual Meeting Procedings. Rosemont, IL, American Academy of Orthopaedic Surgeons; 1992. p. 69.

30. Ries MD, Wiedel JD. Bipolar hip arthroplasty for recurrent dislocation after total hip arthroplasty. A report of three cases. Clin Orthop. 1992;278:121.

31. Paprosky WG. Recurrent dislocation: constrained sockets are the answer. In Opposition. Current Concepts in Joint Replacement. Winter; 2002.

32. Goetz DD, Capello WN, Callaghan JJ, Brown ID, Johnston RC. Salvage of a recurrently dislocating total hip protesis with use of a constrained acetabular component: a retrospective analysis of fifty-six cases. J Bone Joint Surg Am. 1998; 80A:502-9.

33. Anderson MJ, Murray WR, Skinner HB. Constrained acetabular components. J Arthroplasty. 1994;9:17-23.

34. Grigoris P, Grecula MJ, Amstutz HC. Tripolar hip replacement for recurrent prosthetic dislocation. Clin Orthop. 1994;304:148-55.

35. Berry DJ. Unstable total hip arthroplasty: detailed overview. Instructional Course Lectures. 2001;50:265-74.

36. Farizon E, et al. Results with a cementless alumina-coated cup with dual mobility. A twelve-year follow-up study. Int Orthop (SICOT). 1998;22:219-24.

Conflicto de intereses. Los autores no hemos recibido ayuda económica alguna para la realización de este trabajo. Tampoco hemos firmado ningún acuerdo por el que vayamos a recibir beneficios $u$ honorarios por parte de alguna entidad comercial. Por otra parte, ninguna entidad comercial ha pagado ni pagará a fundaciones, instituciones educativas $u$ otras organizaciones sin ánimo de lucro a las que estemos afiliados. 